\title{
Anisotropic Strange Stars through Embedding Technique in Massive Brans-Dicke Gravity
}

\author{
M. Sharif *and Amal Majid ${ }^{\dagger}$ \\ Department of Mathematics, University of the Punjab, \\ Quaid-e-Azam Campus, Lahore-54590, Pakistan.
}

\begin{abstract}
This paper investigates the existence and properties of anisotropic strange quark stars in the context of massive Brans-Dicke theory. The field equations are constructed in Jordan frame by assuming a suitable potential function with MIT bag model. We employ the embedding class-one approach as well as junction conditions to determine the unknown metric functions. Radius of the strange star candidate, LMC $\mathrm{X}-4$, is predicted through its observed mass for different values of the bag constant. We analyze the effects of coupling parameter as well as mass of scalar field on state determinants and execute multiple checks on the stability and viability of the spherical system. It is concluded that the resulting stellar structure is physically viable and stable as it satisfies the energy conditions as well as essential stability criteria.
\end{abstract}

Keywords: Brans-Dicke theory; Anisotropy; Quark stars.

PACS: 04.50.Kd; 04.40.Dg; 97.60.Jd

\section{Introduction}

In the field of astronomy, the observational data of compact stellar structures provide information regarding their mass, rate of rotation and emitted

*msharif.math@pu.edu.pk

†amalmajid89@gmail.com 
radiations. However, in order to understand their internal mechanism and evolutionary process, we rely on analytical methods of relativistic theories. Among compact objects, intriguing nature of neutron stars has motivated researchers to explore their composition, structure and other features. Neutron star has a core of 1 to 3 solar masses $\left(M_{\odot}\right)$ which resists further collapse by counterbalancing the inward pull of gravity through degeneracy pressure of newly generated neutrons. These celestial bodies either exist individually or have a companion to form binary systems. The discovery of neutrons led to the prediction of neutron stars in 1934 [1] but observational evidence came later. This is because neutron stars do not emit enough radiation and are mostly undetectable. Generally, they are spotted as rapidly rotating pulsars which emit radiation at regular intervals ranging from milliseconds to seconds. The first pulsar was discovered in 1967 pulsating for 0.3 seconds after every 1.37 seconds [2]. Some stellar candidates of pulsars include 4U 1820-30, Her X-1, PSR J1903+327, etc.

The study of relativistic objects has revealed that physical properties vary with changes in direction, i.e., they are anisotropic in nature. Anisotropy may occur in both low and high density profiles due to a large number of physical processes such as rotational motion, phase transition or presence of magnetic field or viscous fluid. Ruderman [3] proposed that highly dense system of interacting nuclear matter introduces anisotropy. Since dense cores of stellar objects exhibit extreme nuclear density, anisotropy is one of the dominant features of their intrinsic geometry and evolution. Researchers have explored the effects of anisotropy by considering transverse and radial components of pressure. Herrera and Santos [4] discussed the plausible causes and effects of local anisotropy in self-gravitating systems. Harko and Mak [5] found static interior solutions for anisotropic relativistic objects by considering a specific anisotropy factor. The stability of anisotropic structures including the effects of cosmological constant was explored by Hossein et al. [6]. Paul and Deb [7] formulated physically viable solutions for anisotropic compact stars in hydrostatic equilibrium.

Apart from the three outcomes of collapse (white dwarf, neutron star and black hole), another compact stellar structure is also hypothesized as an end state of inward fall of a neutron star. It is believed that such a cosmic object is composed of strange quark matter which is a favorable state of baryon matter. Witten [8] identified two possibilities of formation of quark matter: the quark-hadron phase transition in the early universe or the transformation of neutron stars into quark stars at extremely high densities. A strange quark 
star is an intermediate stage between black hole and neutron star which has too much mass at its core for the neutrons to hold their individuality but still evades collapse into a black hole. Recent observational estimates of masses and radii of some stars (Her X-1, 4U 1820-30, LMC X-4, etc.) are not consistent with neutron star prototype. Instead they can be treated as suitable candidates for strange quark stars.

The equation of state (EoS) of compact objects (such as neutron and quark stars) is not yet determined despite the existence of several models. The quark star is composed of stable strange quark matter (SQM) (general Wittens conjecture [8]) made of equal number of up, down and strange quarks and is assumed to be the true ground state for the confined hadrons [9]. Interestingly, the neutron star EoS failed to explain the compactness of the compact stellar objects like 4U 1820-30, SAX J 1808.4-3658, 4U 1728-34, Her X-1, RXJ 185635-3754 and PSR 0943+10, etc., whereas SQM EoS (MIT Bag model) [10] has satisfactorily explained the compactness of the stellar candidates. Recent observations of gravitational waves from binary neutron stars collision (GW170817 [11] and GW190425 [12]), have made it possible to estimate the range of masses and thus constraint the mass of neutron and quark stars, determining the MIT bag model as the best approximation for the EoS.

The bag constant $(\mathcal{B})$ appearing in the EoS evaluates the difference between energy density of true (global minimum of energy with stable configuration) and false (local minimum of energy with unstable configuration) vacuum. Increasing the bag constant lowers the quark pressure ultimately affecting the stellar structure. Many people [13] have considered the MIT bag model as an EoS for predicting the interior distribution of quarks in strange stars. Rahaman et al. [14] developed a new interpolating function for calculating the mass of strange stars and explored physical features of a star of radius $9.9 \mathrm{~km}$. A model for a hybrid star composed of normal as well as quark matter was presented by Bhar [15] in the context of Krori and Barura ansatz. Arbañil and Malheiro [16] analyzed the impact of anisotropy on stability as well as equilibrium of quark stars through equations of radial oscillation and hydrostatic equilibrium. The effect of electromagnetic field on anisotropic strange star models has been investigated by employing MIT bag model [17]. Deb et al. [18] studied singularity free solutions representing uncharged as well as charged quark stars with the help of MIT bag model and checked their viability as well as stability. Bhar [19] constructed anisotropic model for strange stars using the condition for embedding class-one and checked it 
for viability and stability.

General relativity (GR) has been accepted as the best fit for describing relativistic structures but suffers from setbacks when defining the current phase of the universe. A natural extension of GR is obtained by including a scalar field that mediates gravity over long ranges. These theories are known as scalar-tensor theories [20] and have extensively been used for discussing different astronomical phenomena. Brans-Dicke (BD) theory, a prototype of scalar-tensor theory, satisfies Mach's principle as well as couples a massless scalar field $\bar{\Phi}$ and metric tensor $g_{\gamma \delta}$ to matter field through a coupling constant $\omega_{B D}$ [21]. In the background of BD theory, $\omega_{B D}$ acts like a tuneable parameter that can take on different values to acquire desirable results.

The value of $\omega_{B D}$ must exceed the value 40,000 in order to satisfy weak field experiments [22] whereas the inflationary model holds for lower values of $\omega_{B D}[23]$. The conflict is resolved by replacing the massless scalar field with a massive scalar field $\Phi$ and adding a self-interacting potential function $V(\Phi)$ in BD theory leading to massive BD (MBD) gravity. The scalar field mass $\left(m_{\Phi}\right)$ leads to a finite range of the scalar field of the order of its compton wavelength $\left(\lambda_{\Phi}\right)$. For $m_{\Phi} \gtrsim 2 \times 10^{-25} \mathrm{GeV}$ (or $\lambda_{\Phi} \lesssim 10^{11} \mathrm{~m}$ ), the solar system observations cannot put stringent restrictions on the BD parameter and all values of $\omega_{B D}$ greater than $-\frac{3}{2}$ are allowed [24]. Like all scalar-tensor theories, MBD can be discussed in both Jordan and Einstein frames based on the nature of coupling (minimal or non-minimal) of scalar field to matter. In Einstein frame, the coupling function $\alpha(\Phi)$ is related to coupling parameter $\omega_{B D}$ in Jordan frame as $\alpha^{2}=\left(2 \omega_{B D}+3\right)^{-1}$.

The slowly and rapidly rotating neutron stars have extensively been discussed in scalar-tensor theories. Sotani [25] studied neutron stars in a massless scalar field and concluded that deviations from GR increase with the increase in mass of celestial objects. Silva et al. [26] discussed the effect of anisotropy on moment of inertia of rotating neutron stars. The structure and properties of slowly rotating neutron stars have also been explored under the influence of a massive scalar field. Doneva and Yazadjiev [27] investigated the dynamics of rapidly rotating neutron stars in the presence of a massive scalar field and concluded that deviations from GR can be large due to larger moment of inertia. Staykov et al. 28 extended this work by considering a self-interacting potential along with a massive scalar field to analyze the behavior of static and slowly rotating neutron stars. Salient features as well as validity of different models for strange quark stars have also been examined in modified theories like $f(R)$ and $f(R, T)$ gravity [29, 30]. Recently, we 
have studied self-gravitating systems by deriving anisotropic extensions of isotropic solutions through gravitational decoupling technique in the context of MBD theory [31].

In this paper, we explore physical attributes of strange stars and discuss their existence in the context of MBD theory. The paper is organized as follows. In section $\mathbf{2}$, we construct a system of field equations and physical variables using MIT bag model. Section 3 gives an overview of junction conditions for a smooth matching between intrinsic and extrinsic geometries. The physical properties, validity and stability are examined in section 4 . In the last section, we summarize our results.

\section{Massive Brans-Dicke Theory and Matter Variables}

Scalar-tensor theories can be represented in Jordan as well as Einstein frames, which are related through a conformal transformation. The action of scalartensor theories in Jordan frame [32] with $G_{0}=1$ is defined as

$$
S=\int \sqrt{-g}\left(\mathcal{R} \Phi-\frac{\omega_{B D}}{\Phi} \nabla^{\gamma} \nabla_{\gamma} \Phi-V(\Phi)+L_{m}\right) d^{4} x
$$

where $g, \mathcal{R}$ and $L_{m}$ represent determinant of the metric tensor, Ricci scalar and matter lagrangian, respectively. The function $V(\Phi)$ completely specifies the scalar-tensor theory. For the present study, we choose

$$
V(\Phi)=\frac{1}{2} m_{\Phi}^{2} \Phi^{2}
$$

Both slowly and rapidly rotating neutron stars have already been studied for this form of potential function [27, 33]. The metric $\hat{g}_{\gamma \delta}$ and scalar field $\hat{\Phi}$ can be obtained for Einstein frame through the transformations $\hat{g}_{\gamma \delta}=\mathcal{A}^{-2}(\Phi) g_{\gamma \delta}$ and $\Phi=\mathcal{A}^{-2}(\hat{\Phi})$. The variation of action (1) with respect to $g_{\gamma \delta}$ and $\Phi$ yields the field equations and evolution equation, respectively, given as

$$
\begin{aligned}
G_{\gamma \delta} & =\frac{1}{\Phi}\left[T_{\gamma \delta}^{(m)}+T_{\gamma \delta}^{\Phi}\right]=\frac{1}{\Phi}\left[T_{\gamma \delta}^{(m)}+\Phi_{, \gamma ; \delta}-g_{\gamma \delta} \square \Phi+\frac{\omega_{B D}}{\Phi}\left(\Phi_{, \gamma} \Phi_{, \delta}\right.\right. \\
& \left.\left.-\frac{g_{\gamma \delta} \Phi_{, \mu} \Phi^{, \mu}}{2}\right)-\frac{V(\Phi) g_{\gamma \delta}}{2}\right]
\end{aligned}
$$




$$
\square \Phi=\frac{T^{(m)}}{3+2 \omega_{B D}}+\frac{1}{3+2 \omega_{B D}}\left(\Phi \frac{d V(\Phi)}{d \Phi}-2 V(\Phi)\right)
$$

where the energy-momentum tensor $T_{\gamma \delta}^{(m)}$ represents the matter distribution and $T^{(m)}$ is its trace with $\square$ being the d'Alembertian operator.

We assume that the static spherical structure of the stellar object is described by the line element

$$
d s^{2}=e^{\nu(r)} d t^{2}-e^{\lambda(r)} d r^{2}-r^{2}\left(d \theta^{2}+\sin ^{2} \theta d \phi^{2}\right),
$$

where $\nu(r)$ and $\lambda(r)$ are metric potentials. Stellar systems are characterized by anisotropic pressure and inhomogeneous energy density which take a dominant part in their evolution. For this purpose, we discuss the physical features of strange stars with anisotropic distribution specified by the following energy-momentum tensor

$$
T_{\gamma \delta}^{(m)}=\left(\rho+p_{\perp}\right) u_{\gamma} u_{\delta}-p_{\perp} g_{\gamma \delta}+\left(p_{r}-p_{\perp}\right) s_{\gamma} s_{\delta},
$$

where $u_{\gamma}=\left(e^{\frac{\nu}{2}}, 0,0,0\right)$ is the 4 -velocity of comoving observer and $s_{\gamma}=$ $\left(0,-e^{\frac{\lambda}{2}}, 0,0\right)$ is a radial 4 -vector. Here, $\rho, p_{r}$ and $p_{\perp}$ represent the energy density, radial and transverse pressures, respectively. Using Eqs.(3) -([6), the field equations are obtained as

$$
\begin{aligned}
\frac{1}{r^{2}}-e^{-\lambda}\left(\frac{1}{r^{2}}-\frac{\lambda^{\prime}}{r}\right) & =\frac{1}{\Phi}\left(\rho+T_{0}^{0 \Phi}\right), \\
-\frac{1}{r^{2}}+e^{-\lambda}\left(\frac{1}{r^{2}}+\frac{\nu^{\prime}}{r}\right) & =\frac{1}{\Phi}\left(p_{r}-T_{1}^{1 \Phi}\right), \\
\frac{e^{-\lambda}}{4}\left(2 \nu^{\prime \prime}+\nu^{\prime 2}-\lambda^{\prime} \nu^{\prime}+2 \frac{\nu^{\prime}-\lambda^{\prime}}{r}\right) & =\frac{1}{\Phi}\left(p_{\perp}-T_{2}^{2 \Phi}\right),
\end{aligned}
$$

where prime denotes differentiation with respect to $r$ and the expressions of $T_{0}^{0 \Phi}, T_{1}^{1 \Phi}$ and $T_{2}^{2 \Phi}$ are given in Appendix A. The wave equation (4) turns out to be

$$
\begin{aligned}
\square \Phi & =-e^{-\lambda}\left[\left(\frac{2}{r}-\frac{\lambda^{\prime}}{2}+\frac{\nu^{\prime}}{2}\right) \Phi^{\prime}(r)+\Phi^{\prime \prime}(r)\right], \\
& =\frac{1}{3+2 \omega_{B D}}\left[T^{(m)}+\left(\Phi \frac{d V(\Phi)}{d \Phi}-2 V(\Phi)\right)\right] .
\end{aligned}
$$


It has been shown that if a symmetric tensor $b_{\gamma \delta}$ satisfies the Gauss-Codazi equations given as

$$
R_{\gamma \delta \mu v}=2 e b_{\gamma[\mu} b_{v] \delta} \quad \text { and } \quad b_{\gamma[\delta ; \mu]}-\Gamma_{\delta \mu}^{\lambda} b_{\gamma \lambda}+\Gamma_{\gamma[\delta}^{\lambda} b_{\mu] \lambda}=0,
$$

the $(n+1)$ dimensional space can be embedded in an $(n+2)$ dimensional pseudo-Euclidean space [34]. Here $e= \pm 1, R_{\gamma \delta \mu v}$ denotes curvature tensor and $b_{\gamma \delta}$ are the co-efficients of second differential form. From the above equation, Eiesland [35] obtained a necessary and sufficient condition for an embedding class-one as

$$
R_{0101} R_{2323}-R_{1212} R_{0303}-R_{1202} R_{1303}=0,
$$

which leads to the following differential equation for the considered metric

$$
\left(\lambda^{\prime}-\nu^{\prime}\right) \nu^{\prime} e^{\lambda}+2\left(1-e^{\lambda}\right) \nu^{\prime \prime}+\nu^{\prime 2}=0 .
$$

The solution of the above equation turns out to be

$$
\lambda(r)=\ln \left(1+B \nu^{\prime 2} e^{\nu}\right),
$$

where $B$ is a constant of integration. Maurya et al. [36] constructed a new class of solutions using the following form of metric potential

$$
\nu(r)=2 r^{2} A+\ln C,
$$

where $A$ and $C$ are positive constants. Using this value in Eq.(14), we have

$$
\lambda(r)=\ln \left(1+A D r^{2} e^{2 A r^{2}}\right),
$$

where $D=16 A B C$ is a constant.

Neutron stars with $M>3 M_{\odot}$ may transform into quark stars which contain up $(u)$, down $(d)$ and strange $(s)$ quark flavors. The matter variables describing the interior configuration of these relativistic stars obey MIT bag EoS. According to the MIT bag model, the quark pressure is stated as

$$
p_{r}=\sum_{f} p^{f}-\mathcal{B}, \quad f=u, d, s,
$$

where $p^{f}$ corresponds to the individual pressure of each quark flavor which is neutralized by the total external bag pressure $\mathcal{B}$, also known as bag constant. The total energy density of deconfined quarks is defined by the bag model as

$$
\rho=\sum_{f} \rho^{f}+\mathcal{B}
$$


where energy density of each flavor $\rho^{f}$ is related to the respective pressure as $\rho^{f}=3 p^{f}$. The EoS of MIT bag model for strange stars is inferred from Eqs.(17) and (18) as

$$
p_{r}=\frac{1}{3}(\rho-4 \mathcal{B}) \text {. }
$$

The simplified form of this EoS has been used in GR and modified theories to examine the features of quark star candidates. In our study, the numerical results of the model have been obtained by taking $\mathcal{B}$ equal to $64 \mathrm{MeV} / \mathrm{fm}^{3}$ and $83 \mathrm{MeV} / \mathrm{fm}^{3}$ which are within the allowed limit [14]. The total mass of a sphere of radius $r$ is evaluated through Misner-Sharp formula as

$$
m=\frac{r}{2}\left(1-e^{-\lambda}\right) .
$$

\subsection{Matching Conditions}

The set of parameters $(A, B, C, D)$ defining the geometry as well as physical properties (such as mass and radius) of anisotropic compact objects can be determined through the smooth matching of interior and exterior spacetimes on the boundary $(\Sigma)$ of the star. The exterior region is taken to be the Schwarzschild spacetime given by

$$
d s^{2}=\left(1-\frac{2 M}{r}\right) d t^{2}-\frac{1}{\left(1-\frac{2 M}{r}\right)} d r^{2}-r^{2}\left(d \theta^{2}+\sin ^{2} \theta d \phi^{2}\right),
$$

where $M$ is the mass. To ensure smoothness and continuity of geometry at the boundary surface, the following conditions must be satisfied at the hypersurface $\Sigma(f=r-R=0, R$ is constant radius $)$

$$
\begin{array}{cl}
\left(d s_{-}^{2}\right)_{\Sigma}=\left(d s_{+}^{2}\right)_{\Sigma}, & \left(K_{i j_{-}}\right)_{\Sigma}=\left(K_{i j_{+}}\right)_{\Sigma} \\
\left(\Phi(r)_{-}\right)_{\Sigma}=\left(\Phi(r)_{+}\right)_{\Sigma}, & \left(\Phi^{\prime}(r)_{-}\right)_{\Sigma}=\left(\Phi^{\prime}(r)_{+}\right)_{\Sigma} .
\end{array}
$$

Here $K_{i j}$ denotes curvature whereas subscripts - and + represent interior and exterior spacetimes, respectively. The continuity of the the first fundamental form $\left(\left[d s^{2}\right]_{\Sigma}=0\right)$ leads to

$$
[H]_{\Sigma} \equiv H\left(r \rightarrow R^{+}\right)-H\left(r \rightarrow R^{-}\right) \equiv H_{R}^{+}-H_{R}^{-},
$$

for any function $H(r)$. The above condition yields $g_{t t}^{-}(R)=g_{t t}^{+}(R)$ and $g_{r r}^{-}(R)=g_{r r}^{+}(R)$. On the other hand, the continuity of the second fundamental form $\left(K_{i j}\right)$ is equivalent to the O'Brien and Synge [37] junction conditions, 
given as

$$
\left[G_{\gamma \delta} r^{\delta}\right]_{\Sigma}=0
$$

where $r_{\gamma}$ is a unit radial vector. Using the above equation and the field equations imply $\left[T_{\gamma \delta} r^{\delta}\right]_{\Sigma}=0$ which leads to $p_{r}(R)=0$. Moreover, the scalar field corresponding to the vacuum Schwarzschild solution is derived using the technique in [38] which comes out to be $\Phi=e^{\left(1-\frac{2 M}{r}\right)}$. We denote the interior and exterior regions by $\mathcal{V}^{-}$and $\mathcal{V}^{+}$, respectively.

The hypersurface is defined by the metric

$$
d s^{2}=d \tau^{2}-R^{2}\left(d \theta^{2}+\sin ^{2} \theta d \phi^{2}\right)
$$

where $\tau$ is the proper time on the boundary. The extrinsic curvature of $\Sigma$ is given by

$$
K_{i j}^{ \pm}=-n_{\gamma}^{ \pm} \frac{\partial^{2} x_{ \pm}^{\gamma}}{\partial \eta^{i} \eta^{j}}-n_{\gamma}^{ \pm} \Gamma_{\delta \mu}^{\gamma} \frac{\partial x_{ \pm}^{\delta}}{\partial \eta^{i}} \frac{\partial x_{ \pm}^{\mu}}{\partial \eta^{j}},
$$

where $\eta^{i}$ are the coordinates defined on the $\Sigma$. Moreover, the components of the four-vector normal $\left(n_{\gamma}^{ \pm}\right)$to the hypersurface are defined in the coordinates $\left(x_{ \pm}^{\gamma}\right)$ of $\mathcal{V}^{ \pm}$as

$$
n_{\gamma}^{ \pm}= \pm \frac{d f}{d x^{\gamma}}\left|g^{\delta \mu} \frac{d f}{d x^{\delta}} \frac{d f}{d x^{\mu}}\right|^{\frac{-1}{2}}
$$

with $n_{\gamma} n^{\gamma}=1$. The unit normal vectors have the following form

$$
n_{\gamma}^{-}=\left(0, e^{\frac{\lambda}{2}}, 0,0\right), \quad n_{\gamma}^{+}=\left(0,\left(1-\frac{2 M}{r}\right)^{\frac{-1}{2}}, 0,0\right) .
$$

Comparing the metrics (51) and (21) with (24), it follows that

$$
\left[\frac{d t}{d \tau}\right]_{\Sigma}=\left[e^{\frac{-\nu}{2}}\right]_{\Sigma}=\left[\left(1-\frac{2 M}{r}\right)^{\frac{-1}{2}}\right]_{\Sigma}, \quad[r]_{\Sigma}=R .
$$

Using Eq.(25), the non-zero components of curvature are calculated as

$$
\begin{aligned}
& K_{00}^{-}=\left[-\frac{e^{-\frac{\lambda}{2}} \nu^{\prime}}{2}\right]_{\Sigma}, \quad K_{22}^{-}=\frac{1}{\sin ^{2}(\theta)} K_{33}^{-}=\left[r e^{-\frac{\lambda}{2}}\right]_{\Sigma} \\
& K_{00}^{+}=\left[-\frac{M}{r^{2}}\left(1-\frac{2 M}{r}\right)^{\frac{-1}{2}}\right]_{\Sigma}, \quad K_{22}^{+}=\frac{1}{\sin ^{2}(\theta)} K_{33}^{+}=\left[r\left(1-\frac{2 M}{r}\right)^{\frac{1}{2}}\right]_{\Sigma} .
\end{aligned}
$$

The junction conditions $\left[K_{22}^{-}\right]_{\Sigma}=\left[K_{22}^{+}\right]_{\Sigma}$ and $[r]_{\Sigma}=R$ yield

$$
e^{-\frac{\lambda(R)}{2}}=\left(1-\frac{2 M}{R}\right)^{\frac{1}{2}}
$$


Substituting the above equation in the matching condition $\left[K_{00}^{-}\right]_{\Sigma}=\left[K_{00}^{+}\right]_{\Sigma}$ gives

$$
\nu^{\prime}(R)=\frac{2 M}{R(R-2 M)} .
$$

Thus, the matching conditions in Eqs.(26)-(28) provide the following relations at the hypersurface

$$
\begin{aligned}
e^{\nu(R)} & =C e^{2 A r^{2}}=1-\frac{2 M}{R}, \\
e^{-\lambda(R)} & =\frac{1}{1+A D R^{2} e^{2 A R^{2}}}=1-\frac{2 M}{R}, \\
\nu^{\prime}(R) & =\frac{2 M}{R(R-2 M)} .
\end{aligned}
$$

Inserting $D=16 A B C$ in the above equations, the deterministic parameters of the system are expressed as

$$
\begin{aligned}
A & =\frac{M}{2 R^{2}(R-2 M)}, \\
B & =\frac{R^{3}}{2 M}, \\
C & =e^{\frac{M}{2 M-R}} \frac{R-2 M}{R}, \\
D & =4 e^{\frac{M}{2 M-R}} .
\end{aligned}
$$

For the metric functions in Eqs.(15) and (16) along with Eqs.(29)-(32), the state variables are expressed in Eqs.(A4)-(A6).

\section{Physical Features of Compact Stars}

The effect of coupling parameter as well as mass of scalar field on stellar structure can now be analyzed through the energy density and radial/transverse pressure components. Since Gravity Probe B experiment provides the lower bound on the mass of scalar field as $m_{\Phi}>10^{-4}$ (in dimensionless units) [24, 27, we take the values of $m_{\Phi}$ as 0.001 and 0.3 . Numerical results have been obtained for $\omega_{B D}=20,25,30$ which are in accordance with the constraints imposed by the solar system observations [24]. The expression of 
scalar field is derived by solving the wave equation numerically with the initial conditions $\Phi(0)=\Phi_{c}=$ constant and $\Phi^{\prime}(0)=0$. The values of $\Phi_{c}$ for different values of $m_{\Phi}, \omega_{B D}$ and $\mathcal{B}$ are given in Tables $\mathbf{1}$ and $\mathbf{2}$. All deductions have been presented graphically for LMC X-4 $\left(M=1.29 M_{\odot}[39]\right)$.

Using the condition $p_{r}(R)=0$, radius as well as physical parameters of the strange star candidate are displayed in Tables $\mathbf{1}$ and $\mathbf{2}$ for $m_{\Phi}=0.001$ and $m_{\Phi}=0.3$, respectively. Here, the subscripts $c$ and $s$ denote that the quantity has been calculated at the center and surface of the star, respectively. For a physically valid solution, the metric potentials must be positive, regular and monotonically increasing functions of the radial coordinate [40]. The potential functions are shown in Figure $\mathbf{1}$ which reveal their regular behavior leading to singularity free system.

The influence of physical variables such as energy density and pressure cannot be neglected in extremely dense strange stars. The behavior of these physical quantities with respect to the radial coordinate is positive throughout and maximum at the center of compact configuration as presented in Figures $\mathbf{2}$ and $\mathbf{3}$ which shows that the core is highly concentrated for the chosen values of the parameters $\left(m_{\Phi}, \omega_{B D}, \mathcal{B}\right)$. The plots also depict the monotonic decreasing trend of energy density and pressure components away from the center of stars leading to a compact profile. Hence, for the considered values of $\mathcal{B}$, the existence of quark stars is ensured for $V(\Phi)=\frac{1}{2} m_{\Phi}^{2} \Phi^{2}$.

The radial and tangential components of pressure give rise to anisotropy within the structure. The anisotropy of pressure, measured as $\Delta=p_{\perp}-p_{r}$, is positive when $p_{\perp}>p_{r}$ and negative otherwise. The positive and increasing behavior of anisotropy suggests that an outward directed repelling force is in play in the interior of stellar models stabilizing the system against gravity. Utilizing Eqs.(A5) and (A6), the anisotropy comes out to be

$$
\begin{aligned}
\Delta & =\frac{\xi \Phi}{r}\left[M^{2} r^{3} \Phi^{2}(r)\left(1-2 e^{\frac{M(r-R)(r+R)}{R^{2}(R-2 M)}}\right)^{2}-r R^{2} \omega_{B D}(2 M-R)\right. \\
& \times\left(2 M r^{2} e^{\frac{M(r-R)(r+R)}{R^{2}(R-2 M)}}+R^{2}(R-2 M)\right) \Phi^{\prime 2}(r)+\Phi(r)\left(R^{4}\left(-(R-2 M)^{2}\right)\right. \\
& \times\left(\Phi^{\prime}(r)-r \Phi^{\prime \prime}(r)\right)-2 M r^{2} e^{\frac{M(r-R)(r+R)}{R^{2}(R-2 M)}}\left(\left(M r^{2}-4 M R^{2}+2 R^{3}\right) \Phi^{\prime}(r)\right. \\
& \left.\left.\left.+r R^{2}(2 M-R) \Phi^{\prime \prime}(r)\right)\right)\right] .
\end{aligned}
$$

Figure 4 indicates that the behavior of anisotropy is acceptable for the se- 
Table 1: Physical parameters of LMC X-4 with $m_{\Phi}=0.001$ for different values of $\omega_{B D}$ and $\mathcal{B}$.

\begin{tabular}{|c|c|c|c|c|c|}
\hline \multicolumn{6}{|c|}{$\mathcal{B}=64 M e V / f^{3}$} \\
\hline$\omega_{B D}$ & $\overline{\Phi_{c}}$ & $\begin{array}{c}\text { Predicted } \\
\text { Radius }(\mathrm{km})\end{array}$ & $\rho_{c}\left(\mathrm{gm} / \mathrm{cm}^{3}\right)$ & $\rho_{s}\left(\mathrm{gm} / \mathrm{cm}^{3}\right)$ & $p_{c}\left(\right.$ dyne $\left./ \mathrm{cm}^{2}\right)$ \\
\hline 20 & 0.0204 & $8.3141_{-0.3285}^{+0.3293}$ & $6.6437 \times 10^{14}$ & $4.6155 \times 10^{14}$ & $7.1544 \times 10^{34}$ \\
\hline 25 & 003055 & $9.6173_{-0.3847}^{+0.3863}$ & $6.2865 \times 10^{14}$ & $4.5450 \times 10^{14}$ & $5.6021 \times 10^{34}$ \\
\hline 30 & 0.04445 & $10.9515_{-0.446}^{+0.449}$ & $6.1019 \times 10^{14}$ & $4.6035 \times 10^{14}$ & $4.8650 \times 10^{34}$ \\
\hline GR limit & & 5.54 & $9.498 \times 10^{16}$ & $6.823 \times 10^{16}$ & $3.540 \times 10^{36}$ \\
\hline \multicolumn{6}{|c|}{$\mathcal{B}=83 \mathrm{MeV} / \mathrm{fm}^{3}$} \\
\hline$\omega_{B D}$ & $\overline{\Phi_{c}}$ & $\begin{array}{c}\text { Predicted } \\
\text { Radius }(\mathrm{km})\end{array}$ & $\rho_{c}\left(\mathrm{gm} / \mathrm{cm}^{3}\right)$ & $\rho_{s}\left(\mathrm{gm} / \mathrm{cm}^{3}\right)$ & $p_{c}\left(\right.$ dyne $\left./ \mathrm{cm}^{2}\right)$ \\
\hline 20 & 00264 & $8.3397_{-0.3315}^{+0.3582}$ & $8.5180 \times 10^{14}$ & $5.9133 \times 10^{14}$ & $9.0542 \times 10^{34}$ \\
\hline 25 & 0.0399 & $9.6669_{-0.3908}^{+0.3931}$ & $8.1568 \times 10^{14}$ & $5.9266 \times 10^{14}$ & $7.1989 \times 10^{34}$ \\
\hline 30 & 0.0578 & $11.0422_{-0.4577}^{+0.4622}$ & $7.9254 \times 10^{14}$ & $5.8838 \times 10^{14}$ & $6.3199 \times 10^{34}$ \\
\hline GR limit & & 5.55 & $9.498 \times 10^{16}$ & $6.823 \times 10^{16}$ & $3.564 \times 10^{36}$ \\
\hline
\end{tabular}

Table 2: Physical parameters of LMC X-4 with $m_{\Phi}=0.3$ for different values of $\omega_{B D}$ and $\mathcal{B}$.

\begin{tabular}{|c|c|c|c|c|c|}
\hline \multicolumn{6}{|c|}{$\mathcal{B}=64 \mathrm{MeV} / \mathrm{fm}^{3}$} \\
\hline$\omega_{B D}$ & $\Phi_{c}$ & $\begin{array}{c}\text { Predicted } \\
\text { Radius }(\mathrm{km})\end{array}$ & $\rho_{c}\left(\mathrm{gm} / \mathrm{cm}^{3}\right)$ & $\rho_{s}\left(\mathrm{gm} / \mathrm{cm}^{3}\right)$ & $p_{c}\left(\right.$ dyne $\left./ \mathrm{cm}^{2}\right)$ \\
\hline 20 & 0.0204 & $8.3141_{-0.3285}^{+0.3293}$ & $6.7762 \times 10^{14}$ & $4.7467 \times 10^{14}$ & $7.1905 \times 10^{34}$ \\
\hline 25 & 0.03055 & $9.6173_{-0.3847}^{+0.3863}$ & $6.6665 \times 10^{14}$ & $4.9018 \times 10^{14}$ & $5.8065 \times 10^{34}$ \\
\hline 30 & 0.04555 & $10.9515_{-0.446}^{+0.449}$ & $6.7427 \times 10^{14}$ & $5.1681 \times 10^{14}$ & $4.9215 \times 10^{34}$ \\
\hline \multicolumn{6}{|c|}{$\mathcal{B}=83 \mathrm{MeV} / \mathrm{fm}^{3}$} \\
\hline$\omega_{B D}$ & $\Phi_{c}$ & $\begin{array}{c}\text { Predicted } \\
\text { Radius }(\mathrm{km})\end{array}$ & $\rho_{c}\left(\mathrm{gm} / \mathrm{cm}^{3}\right)$ & $\rho_{s}\left(\mathrm{gm} / \mathrm{cm}^{3}\right)$ & $p_{c}\left(\right.$ dyne $\left./ \mathrm{cm}^{2}\right)$ \\
\hline 20 & 0.0264 & $8.3397_{-0.3315}^{+0.3582}$ & $8.7829 \times 10^{14}$ & $6.1661 \times 10^{14}$ & $9.1757 \times 10^{34}$ \\
\hline 25 & 0.0399 & $9.6669_{-0.3908}^{+0.3931}$ & $8.5916 \times 10^{14}$ & $6.3427 \times 10^{14}$ & $7.2289 \times 10^{34}$ \\
\hline 30 & 0.0578 & $11.0422_{-0.4577}^{+0.4622}$ & $8.8271 \times 10^{14}$ & $6.9153 \times 10^{14}$ & $6.0806 \times 10^{34}$ \\
\hline
\end{tabular}



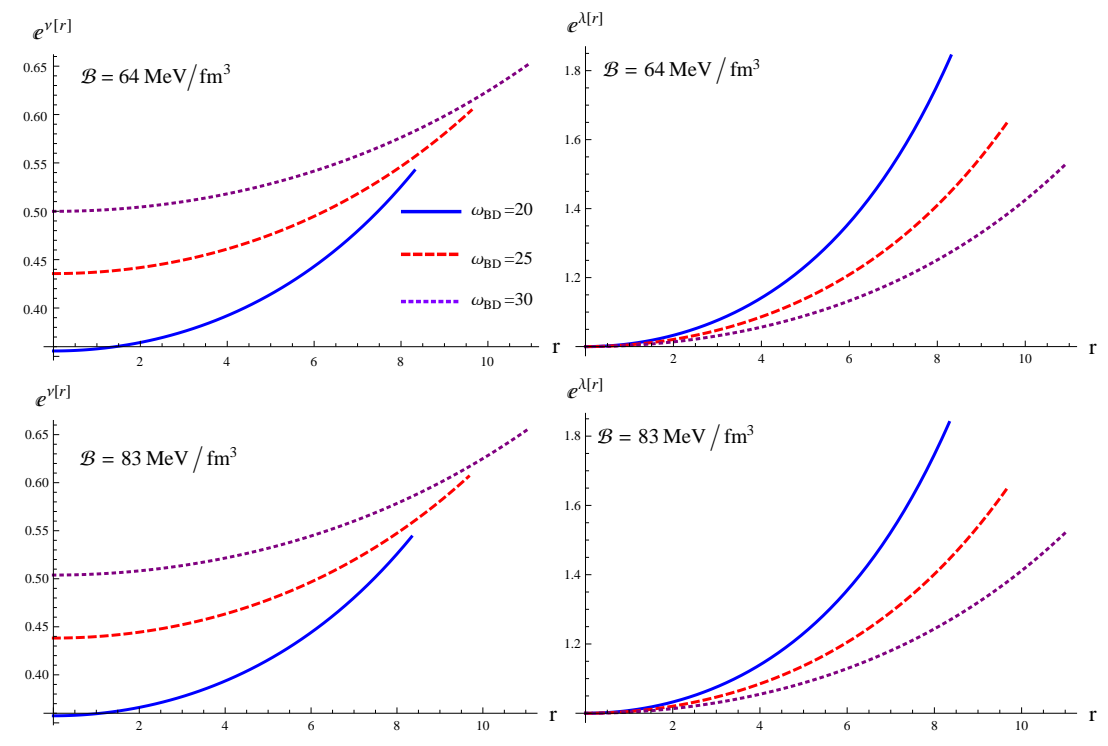

Figure 1: Plots of metric potentials for massive scalar field versus radial coordinate.
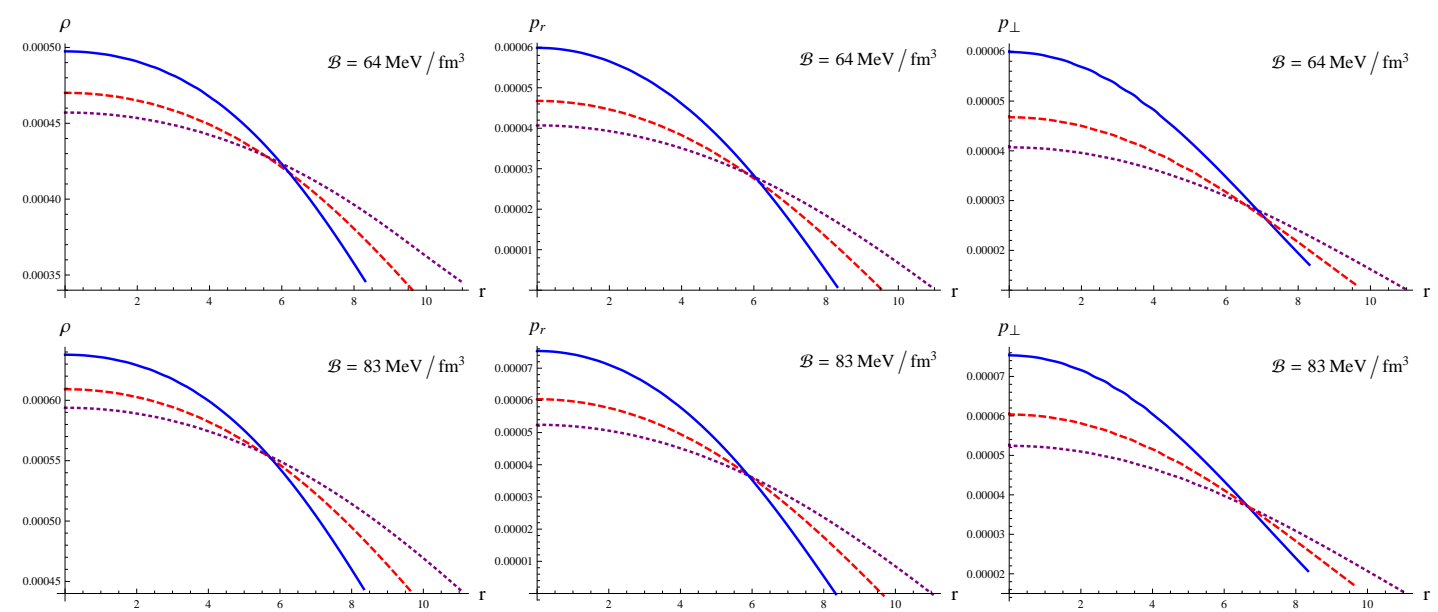

Figure 2: Effective energy density, effective radial/transverse pressure as functions of $r$ with $m_{\Phi}=0.001$. 

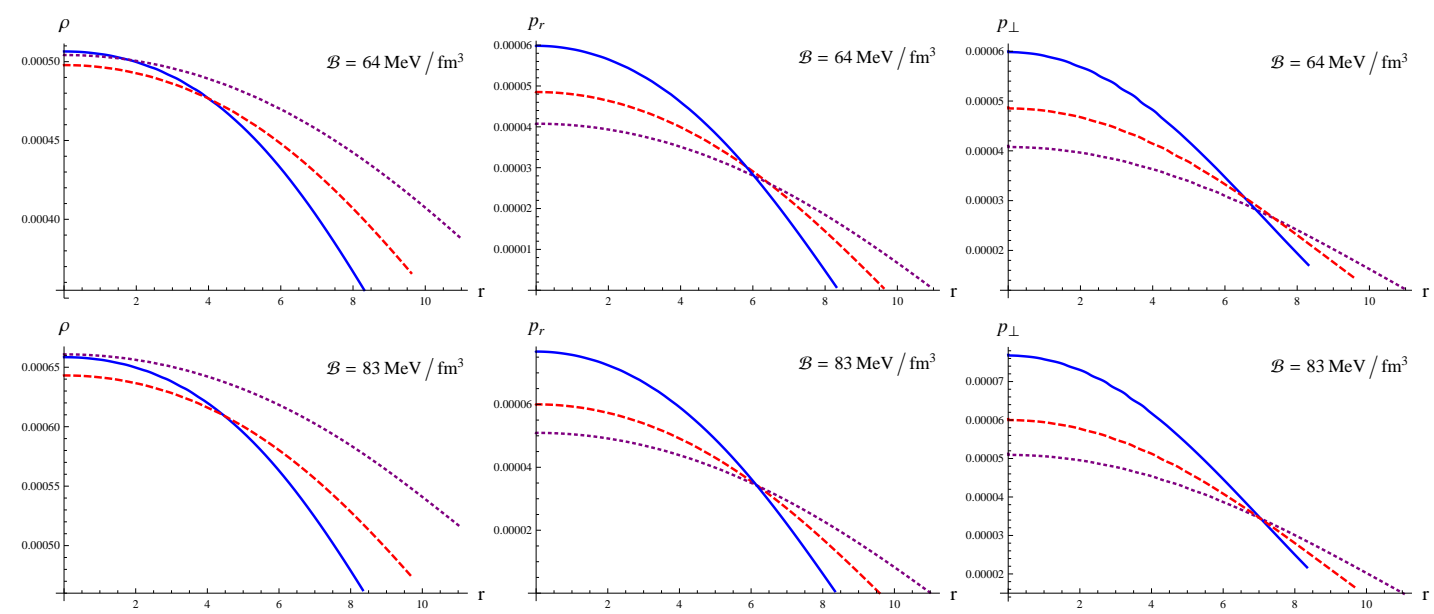

Figure 3: Effective energy density, effective radial/transverse pressure as functions of $r$ with $m_{\Phi}=0.3$.
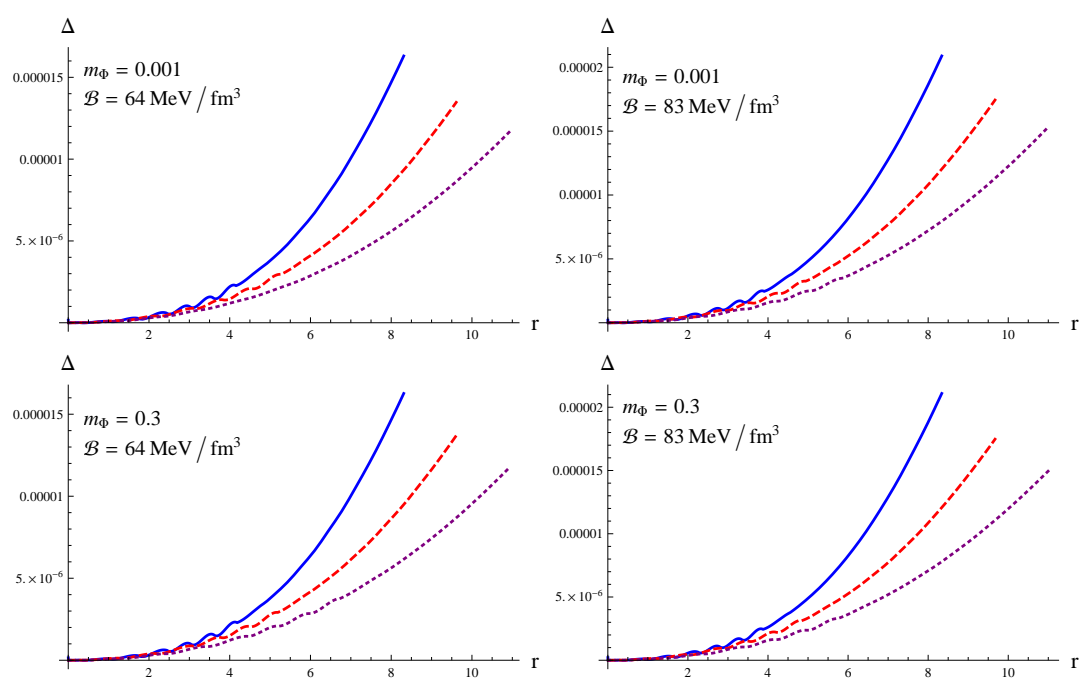

Figure 4: Variation of effective anisotropy as a function of $r$. 
lected model.

\subsection{Energy Conditions}

A configuration is said to be realistic if it satisfies all four energy conditions, i.e., null (NEC), weak (WEC), strong (SEC) and dominant (DEC). These conditions are evaluated in terms of effective energy density and effective components of pressure in the presence of scalar field as [41]

$$
\begin{array}{ll}
\text { NEC: } & \rho \geq 0, \\
\text { WEC: } & \rho+p_{r} \geq 0, \quad \rho+p_{\perp} \geq 0, \\
\text { SEC: } \quad \rho+p_{r}+2 p_{\perp} \geq 0, & \\
\text { DEC: } \quad \rho-p_{r} \geq 0, \quad \rho-p_{\perp} \geq 0 .
\end{array}
$$

Figures 2 and $\mathbf{3}$ depict positive behavior of $\rho, p_{r}$ and $p_{\perp}$ throughout the stellar structure, the first three conditions are readily satisfied. The plot of DEC in Figure $\mathbf{5}$ is positive at each point within the stellar structure. Hence, all energy conditions are satisfied which validate the model for the chosen values of $m_{\Phi}, \mathcal{B}$ and $\omega_{B D}$.

\subsection{Effective Mass, Compactness and Redshift}

The size and mass are two inter-related observable features of a compact object. The effective mass for the current structure is calculated via Eq.(20) as

$$
m(r)=\frac{r}{2}\left[\frac{2 M r^{2} e^{\frac{M\left(R^{2}-r^{2}\right)}{R^{2}(2 M-R)}}}{R^{2}(R-2 M)+2 M r^{2} e^{\frac{M\left(R^{2}-r^{2}\right)}{R^{2}(2 M-R)}}}\right],
$$

which is dependent on the radius of celestial body. Figure 6 shows a decrease in mass for a larger value of $\mathcal{B}$. The compactness function is the ratio of mass to radius given as

$$
u(r)=\frac{m(r)}{r}=\frac{1}{2}\left[\frac{2 M r^{2} e^{\frac{M\left(R^{2}-r^{2}\right)}{R^{2}(2 M-R)}}}{R^{2}(R-2 M)+2 M r^{2} e^{\frac{M\left(R^{2}-r^{2}\right)}{R^{2}(2 M-R)}}}\right] .
$$

Figure $\mathbf{6}$ displays the compactness factor as a monotonic increasing function with respect to the radial coordinate. The values attained by the function 

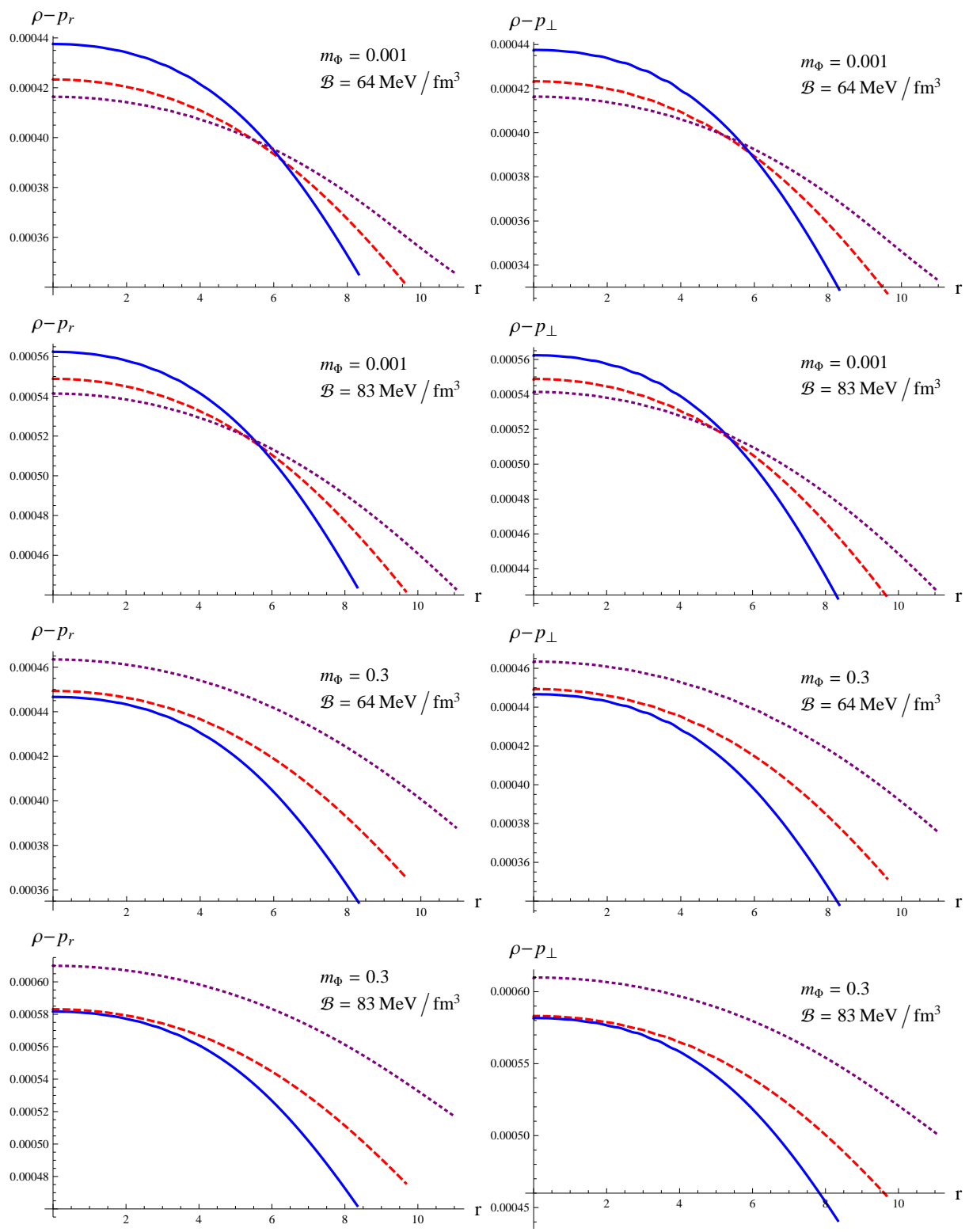

Figure 5: Dominant energy condition plotted against the radial coordinate. 

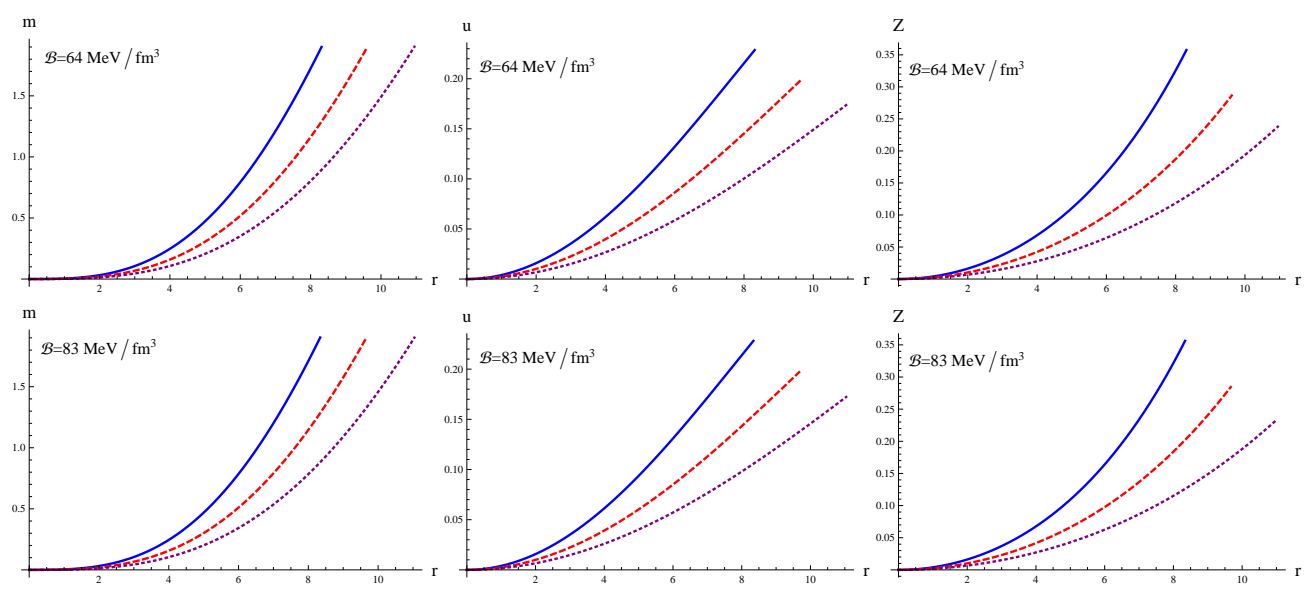

Figure 6: Plots of relation between mass, compactness factor and redshift against radial coordinate.

adhere to the upper limit $\frac{m}{R}<\frac{4}{9}$, proposed by Buchdal [42] for both values of bag constant. Further, the gravitational redshift is a measure of the force exerted on light as a consequence of strong gravity. The relativistic effect can be measured from the X-ray spectrum of the cosmic object using the compactness factor which is defined as

$$
Z=\frac{1}{\sqrt{1-2 u(r)}}-1
$$

leading to the following expression

$$
Z=-1+\sqrt{1+\frac{2 M r^{2} e^{\frac{M\left(R^{2}-r^{2}\right)}{R^{2}(2 M-R)}}}{R^{2}(R-2 M)}}
$$

Figure 6 exhibits the redshift as an increasing function of radial coordinate. We would like to mention here that the surface redshift for the stellar candidate is consistent with the limit for relativistic stars $(Z<5.211)$ [43].

\subsection{Stability of Stellar System}

In this section, we examine stability of the anisotropic setup. It is crucial for a stable anisotropic system that the speed of sound is less than that of 

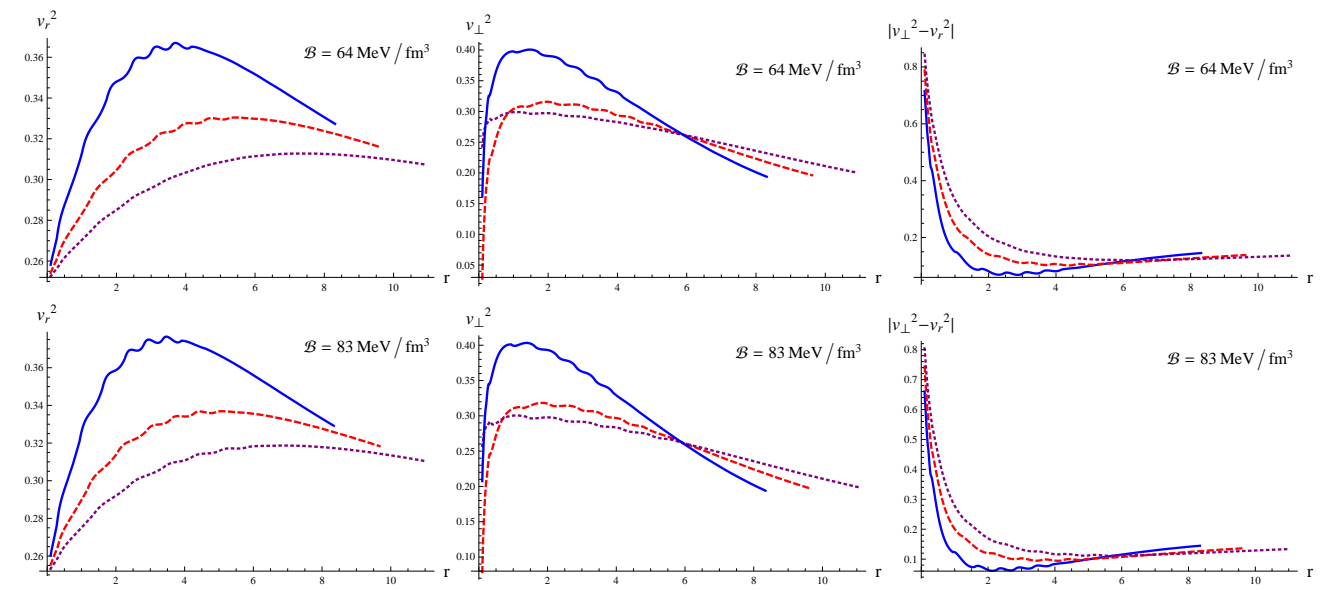

Figure 7: Variation of radial velocity, tangential velocity and $\left|v_{\perp}^{2}-v_{r}^{2}\right|$ with respect to radial coordinate with $m_{\Phi}=0.001$.

light, i.e., $0<v_{r}^{2}<1$ and $0<v_{\perp}^{2}<1$, where $v_{r}$ and $v_{\perp}$ are the radial and tangential components of speed expressed, respectively, as

$$
v_{r}^{2}=\frac{d p_{r}}{d \rho}, \quad v_{\perp}^{2}=\frac{d p_{\perp}}{d \rho} .
$$

This criterion is known as the condition of causality [44. The stability of a system can also be verified through Herrera's cracking approach [45]. Cracking occurs when inward directed radial forces of a perturbed system change direction for some value of radial coordinate. According to this scheme, a region free from cracking is stable when $0<\left|v_{\perp}^{2}-v_{r}^{2}\right|<1$. One of the interesting features of this method is that cracking is closely related to changes in local anisotropy. Figures $\mathbf{7}$ and $\mathbf{8}$ shows that anisotropic distribution agrees with the causality condition as well as cracking approach in the framework of MBD theory.

Another commonly used tool to examine the stability of relativistic spherical systems is adiabatic index. This indicates stiffness of the EoS for a specific energy density by connecting the EoS with the internal structure of the sphere. Chandrasekhar [46] studied the dynamical stability of relativistic stars against infinitesimal radial adiabatic perturbation. Heintzmann and Hillebrandt [4] found that an anisotropic compact object will achieve stability if the adiabatic index is greater than $\frac{4}{3}$ everywhere inside the con- 

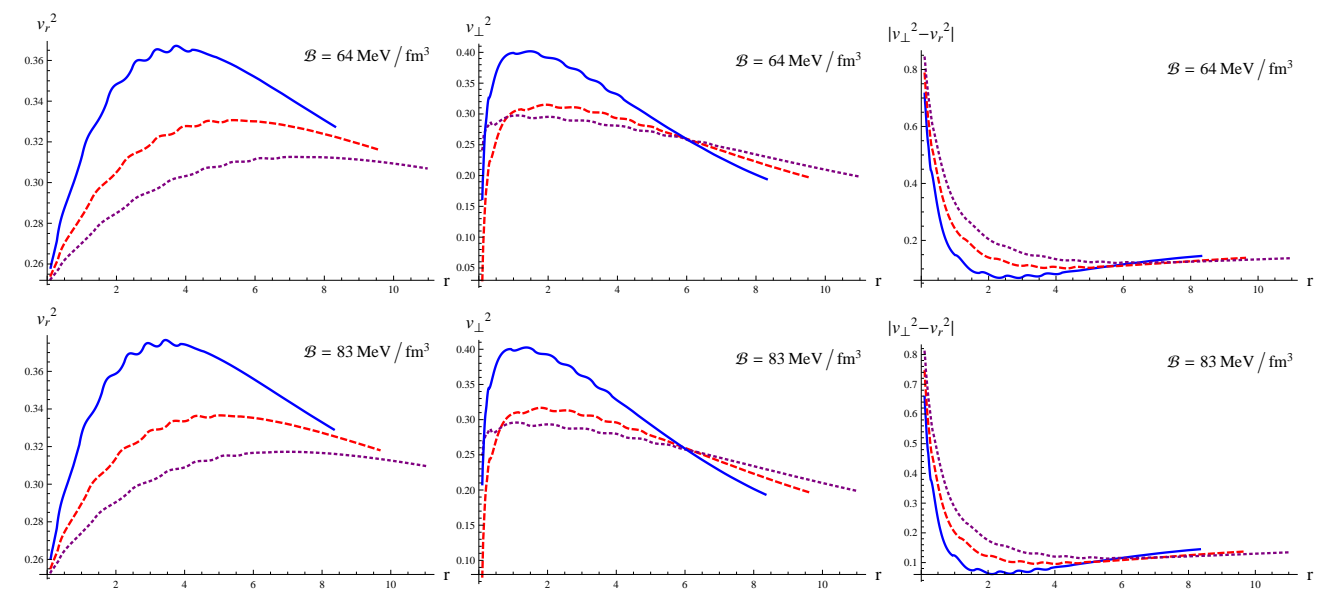

Figure 8: Variation of radial velocity, tangential velocity and $\left|v_{\perp}^{2}-v_{r}^{2}\right|$ with respect to radial coordinate with $m_{\Phi}=0.3$.

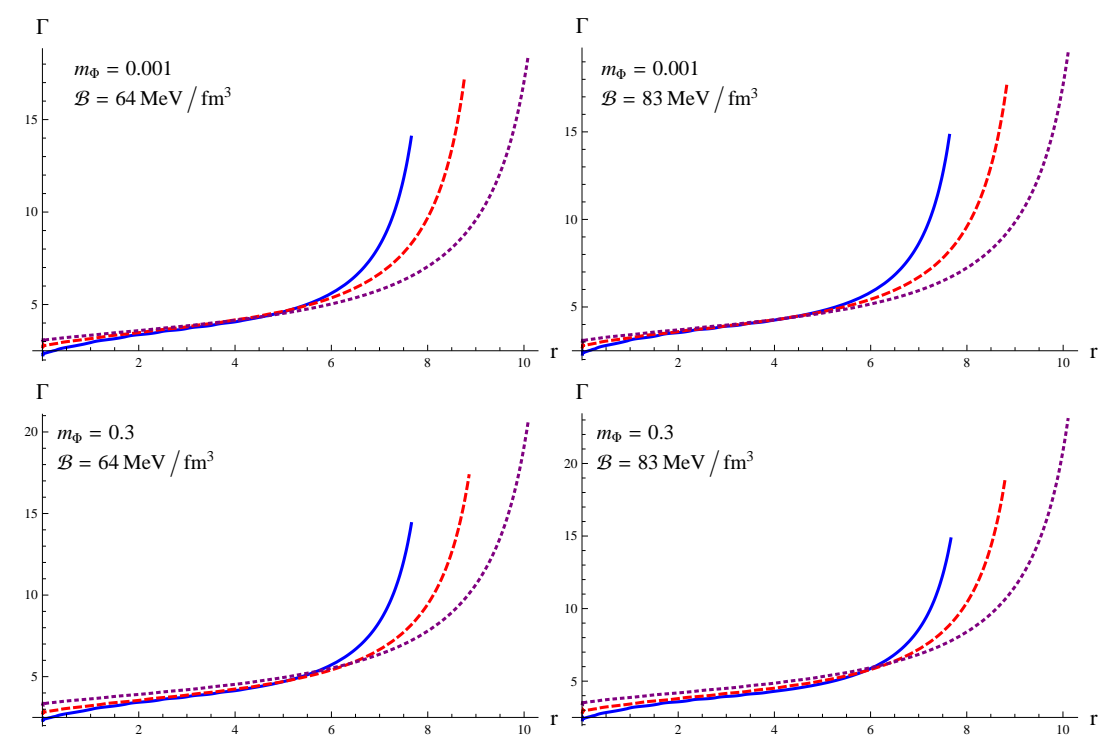

Figure 9: Plots of adiabatic index versus $r$. 
figuration. The expression for adiabatic index for our system is given by

$$
\Gamma=\frac{p_{r}+\rho}{p_{r}} \frac{d p_{r}}{d \rho}=\frac{p_{r}+\rho}{p_{r}} v_{r}^{2}
$$

The graphical analysis of adiabatic index in the presence of a scalar field can be seen in Figure 9. The value of this index is more than $\frac{4}{3}$ for all stars which is in agreement with the constraint [47]. Hence, the stellar structure is stable for the considered values of MBD parameters.

\section{Concluding Remarks}

In the field of astrophysics, the evolution of stellar models and their remnants have been examined by many researchers. Strange quark stars are hypothesized to emerge from the collapse of neutron stars and are composed of three quark flavors. These celestial bodies are highly dense compact objects whose structures depend on central and surface densities. This work investigates the possible existence of anisotropic hypothetical objects in the background of MBD theory. We have formulated the field equations in Jordan frame by selecting $V(\Phi)=\frac{1}{2} m_{\Phi}^{2} \Phi^{2}, m_{\Phi}=0.001,0.3$ and $\omega_{B D}=20,25,30$. A solution to the field equations has been generated by assuming a well-behaved metric potential and embedding class-one condition with MIT bag model. Utilizing the matching conditions at the boundary of the star, the effective energy density and effective pressure components have been expressed in terms of mass and radius. The structure has finally been determined by evaluating the constants $(A, B, C, D)$ through the observed mass and predicted radius of LMC X-4. The effect of bag constant on stability and viability of the system has been checked through various criteria by taking into account two values of $\mathcal{B}$ as $64 \mathrm{MeV} / \mathrm{fm}^{3}$ and $83 \mathrm{MeV} / \mathrm{fm}^{3}$.

Tables $\mathbf{1}$ and $\mathbf{2}$ indicate that predicted radius increases while Figure $\mathbf{6}$ shows a decrease in mass as the bag constant increases in the presence of massive scalar field. This implies that the quark star becomes more dense with increase in the bag constant. Moreover, higher values of scalar field mass lead to more dense stellar systems as indicated in Tables $\mathbf{1}$ and $\mathbf{2}$. The behavior of physical attributes of the stellar candidates has also been explored graphically. It is observed that energy density and pressure components are finite at the center and monotonically decrease towards the surface. The regular behavior of state variables indicates that the system has no singularity. 
We have established that the interior of star consists of normal matter as all energy conditions are satisfied for the considered values of the parameters as well as the bag constant.

The positive trend of anisotropy confirms the repelling force required to save the star from further collapse. The graphical analysis of redshift parameter which depicts that as the radius of the star increases, the amount of redshift (complying with the limit $Z<5.211$ [43]) decreases. We have also calculated the compactness factor and the mass-radius ratio which are in agreement with the Buchdahl criterion [42]. The graphical representation of redshift and compactness factor of the stellar model shows increasing behavior with a decrease in radius. Hence, a more dense star exerts additional force on light leading to greater redshift. Finally, we have checked the stability conditions for the prototype stellar model using three approaches. All these imply stability of the system coupled to a massive scalar field. However, plots of $v_{r}$ and $v_{\perp}$ represent smooth behavior for increasing values of $\omega_{B D}$ as shown in Figures $\mathbf{7}$ and $\mathbf{8}$. Hence, the celestial object is more stable for larger values of coupling parameter. We conclude that the cosmic structure governed by MIT bag model in the framework of MBD is consistent with all the critical requirements and can be treated as a viable and stable model. It is worthwhile to mention here that our results are consistent with $f(R, T)$ theory [30]. All our results reduce to GR for $\omega_{B D} \rightarrow \infty$.

The stellar model has also been constructed in GR with the help of metric potentials in Eqs.(15) and (16) to highlight the effect of massive scalar field. It describes a star with estimated radii of $5.54 \mathrm{~km}$ and $5.55 \mathrm{~km}$ corresponding to $\mathcal{B}=64 \mathrm{MeV} / \mathrm{fm}^{3}$ and $83 \mathrm{MeV} / \mathrm{fm}^{3}$, respectively. Thus, GR predicts smaller stars with increased central $\left(9.498 \times 10^{16} \mathrm{gm} / \mathrm{cm}^{3}\right)$ and surface $\left(6.823 \times 10^{16} \mathrm{gm} / \mathrm{cm}^{3}\right)$ densities as compared to the MBD stellar model. Moreover, the relativistic model in GR has also increased central pressure $\left(3.540 \times 10^{36}\right.$ dyne $/ \mathrm{cm}^{2}, 3.5640 \times 10^{36}$ dyne $\left./ \mathrm{cm}^{2}\right)$ for both values of the bag constant.

\section{Appendix A}

The components of $T_{\delta}^{\gamma \Phi}$ are obtained as

$$
T_{0}^{0 \Phi}=e^{-\lambda}\left[\Phi^{\prime \prime}+\left(\frac{2}{r}-\frac{\lambda^{\prime}}{2}\right) \Phi^{\prime}+\frac{\omega_{B D}}{2 \Phi} \Phi^{\prime 2}-e^{\lambda} \frac{V(\Phi)}{2}\right],
$$




$$
\begin{aligned}
& \left.T_{1}^{1 \Phi}=e^{-\lambda}\left[\left(\frac{2}{r}+\frac{\nu^{\prime}}{2}\right) \Phi^{\prime}-\frac{\omega_{B D}}{2 \Phi} \Phi^{\prime 2}-e^{\lambda} \frac{V(\Phi)}{2}\right)\right] \\
& T_{2}^{2 \Phi}=e^{-\lambda}\left[\Phi^{\prime \prime}+\left(\frac{1}{r}-\frac{\lambda^{\prime}}{2}+\frac{\nu^{\prime}}{2}\right) \Phi^{\prime}+\frac{\omega_{B D}}{2 \Phi} \Phi^{\prime 2}-e^{\lambda} \frac{V(\Phi)}{2}\right]
\end{aligned}
$$

Energy density and pressure components take the following form

$$
\begin{aligned}
\rho & =\frac{1}{2 r^{2}}\left\{\xi^{2} \Phi^{2}\left[2 R^{2}(2 M-R)\left(M\left(r^{2}-2 R^{2}\right)+R^{3}\right)\left(r \Phi^{\prime}(r)+2 \Phi(r)\right)\right]\right. \\
& -\xi\left[r^{2} R^{2} \omega_{B D}(R-2 M) \Phi^{\prime 2}(r)+2 r \Phi(r)\left(\left(R^{3}-M\left(r^{2}+2 R^{2}\right)\right) \Phi^{\prime}(r)\right.\right. \\
& \left.\left.+r R^{2}(R-2 M) \Phi^{\prime \prime}(r)\right)-2 \Phi^{2}(r)\left(2 M(r-R)(r+R)+R^{3}\right)\right] \\
& \left.+r^{2} V(\Phi)+2 \Phi(r)\right\}, \\
p_{r} & =\frac{\xi}{4 r^{2}} r^{2} R^{2} \omega_{B D}(2 M-R) \Phi^{\prime 2}(r)+2 \Phi^{2}(r)\left(2 M(r-R)(r+R)+R^{3}\right) \\
& +r \Phi(r)\left(\left(2 M(r-R)(r+R)+R^{3}\right) \Phi^{\prime}(r)+r R^{2}(2 M-R) \Phi^{\prime \prime}(r)\right) \\
& +\frac{\xi^{2} \Phi^{2}}{4 r}\left(R^{2}(2 M-R)\left(M\left(r^{2}-2 R^{2}\right)+R^{3}\right)\left(r \Phi^{\prime}(r)+2 \Phi(r)\right)\right)-\mathcal{B},(\mathrm{A} 5) \\
& =\frac{\Phi \xi}{4 r^{2}}\left[( M ( r ^ { 2 } - 2 R ^ { 2 } ) + R ^ { 3 } ) \left(2 \Phi(r)\left(2 M(r-R)(r+R)+R^{3}\right)\right.\right. \\
& \left.\left.+3 r R^{2}(R-2 M) \Phi^{\prime}(r)\right)\right]+\xi\left[3 r^{2} R^{2} \omega_{B D}(R-2 M) \Phi^{\prime 2}(r)+r \Phi(r)\right. \\
& \times\left(\left(-2 M r^{2}+14 M R^{2}-7 R^{3}\right) \Phi^{\prime}(r)+3 r R^{2}(R-2 M) \Phi^{\prime \prime}(r)\right)-2 \Phi^{2}(r) \\
& \left.\times\left(2 M\left(r^{2}-3 R^{2}\right)+3 R^{3}\right)\right]-\mathcal{B}+\frac{\Phi(r)}{p^{2}},
\end{aligned}
$$

where $\xi=\frac{\Phi^{-1}(r)}{\left(2 M r^{2} e^{\frac{M(r-R)(r+R)}{R^{2}(R-2 M)}}+R^{2}(R-2 M)\right)}$.

\section{References}

[1] Baade, W. and Zwicky, F.: Phys. Rev. 46(1934)76.

[2] Hewish, A. et al.: Nature 217(1968)709.

[3] Ruderman, A.: Annu. Rev. Astron. Astrophs. 10(1972)427.

[4] Herrera, L. and Santos, N.O.: Phys. Reports 286(1997)53.

[5] Harko, T. and Mak, M.K.: Annalen Phys. 11(2002)3. 
[6] Hossein, S.K.M. et al.: Int. J. Mod. Phys. D 21(2012)1250088.

[7] Paul, B.C. and Deb, R.: Astrophys. Space Sci. 354(2014)421.

[8] Witten, E.: Phys. Rev. D 30(1984)272.

[9] Alcock, C. and Olinto, A.V.: Annu. Rev. Nucl. Part. Sci. 38(1988)161; Madsen, J.: Lect. Notes Phys. 516(1999)162.

[10] Bordbar, G.H. and Peivand, A.R.: Res. Astron. Astrophys. 11(2011)851.

[11] Abbott, B.P. et al.: Phys. Rev. Lett. 119(2017)161101.

[12] The LIGO Scientific Collaboration, the Virgo Collaboration, Abbott, B.P. et al: Astrophys. J. Lett. 892(2020)L3.

[13] Haensel, P., Zdunik, J.L. and Schaffer, R.: Astron. Astrophys. 160(1986)121; Cheng, K.S., Dai, Z.G. and Lu, T.: Int. J. Mod. Phys. D 7 (1998)139; Harko, T. and Mak, M.K.: Chin. J. Astron. Astrophys. 2(2002)248.

[14] Rahaman, F. et al.: Eur. Phys. J. C 74(2014)3126.

[15] Bhar, P.: Astrophys. Space Sci. 357(2015)46.

[16] Arbañil, J.D.V and Malheiro, M.: AIP Conf. Proc. 1693(2015)030007.

[17] Maurya, S.K. et al.: Eur. Phys. J. C 75(2015)389; Maurya, S.K. et al.: Astrophys. Space Sci. 361(2016)163; Murad, M.H.: Astrophys. Space Sci. 361(2016)20.

[18] Deb, D. et al.: Ann. Phys. 387(2017)239; Eur. Phys. J. C 78(2018)465.

[19] Bhar, P.: Eur. Phys. J. C 79(2019)138.

[20] Will, C.M.: Theory and Experiment in Gravitational Physics (Cambridge University Press, 1993).

[21] Brans, C. and Dicke, R.H.: Phys. Rev. 124(1961)3.

[22] Will, C.M.: Living Rev. Rel. 4(2001)4. 
[23] Weinberg, E.J.: Phys. Rev. D 40(1989)3950.

[24] Perivolaropoulos, L.: Phys. Rev. D 81(2010)047501.

[25] Sotani, H.: Phys. Rev. D 86(2012)124036.

[26] Silva, H.O. et al.: Class. Quantum Grav. 32(2015)145008.

[27] Doneva, D.D. and Yazadjiev, S.S.: J. Cosmol. Astropart. Phys. 11(2016)019.

[28] Staykov, K.V.: Eur. Phys. J. C 78(2018)586.

[29] Astashenok, A.V.: Int. J. Mod. Phys.: Conf. Series 41(2016)1660130; Sharif, M. and Waseem, A.: Eur. Phys. J. C 78(2018)868; Deb, D. et al.: arXiv:1812.11736.

[30] Maurya, S.K. et al.: Phys. Rev. D 100(2019)044014.

[31] Sharif, M. and Majid, A.: Astrophys. Space Sci. 365(2020)42.

[32] Khoury, J. and Weltman, A.: Phys. Rev. D 69(2004)044026.

[33] Yazadjiev, S.S., Doneva, D.D. and Popchev, D.: Phys. Rev. D 93(2016)084038.

[34] Eisenhart, L.P.: Riemannian Geometry (Princeton University Press, 1925).

[35] Eiesland, J.: Trans. Am. Math. Soc. 27(1925)213.

[36] Maurya, S.K. et al.: Eur. Phys. J. C 76(2016)266.

[37] O'Brien, S. and Synge, J.L.: Commun. Dublin Inst. Adv. Stud. A $\mathbf{9}(1952)$.

[38] Bruckman, W.F. and Kazes, E.: Phys. Rev. D 16(1977)2.

[39] Rawls, M.L. et al.: Astrophys. J. 730(2011)25.

[40] Lake, K.: Phys. Rev. D 67(2003)104015.

[41] Fujii, Y. and Maeda, K.: The Scalar-Tensor Theory of Gravitation (Cambridge University Press, 2003). 
[42] Buchdahl, H.A.: Phys. Rev. D 116(1959)1027.

[43] Ivanov, B.V.: Phys. Rev. D 65(2002)104011.

[44] Abreu, H., Hernandez, H. and Nunez, L.A.: Class. Quantum Gravit. 24(2007)4631.

[45] Herrera, L.: Phys. Lett. A 165(1992)206.

[46] Chandrasekhar, S.: Astrophys. J. 140(1964)417; Phys. Rev. Lett. $12(1964) 114$.

[47] Heintzmann, H. and Hillebrandt, W.: Astron. Astrophys. 24( 1975)51. 